\title{
Reliability Research on Hydraulic System of Civil Aircraft
}

\author{
Jiangtao Chen ${ }^{1}$, Rui Liu ${ }^{1}$ \\ ${ }^{1}$ China Aviation Industry General Aircraft Institute Co. Ltd., Zhuhai, Guangdong, China \\ ${ }^{*}$ Corresponding Author:Jiangtao Chen \\ ${ }^{\text {a }}$ email
}

Keywords:Reliability Research, Hydraulic System, Civil Aircraft

\begin{abstract}
The civil aviation transportation industry of our country is growing explosively. The hydraulic system of civil aircrafts is crucial to the whole civil aircraft. This paper focuses on the hydraulic system of civil aircraft, which includes the Relevant Parameters of Reliability, the prediction and the test of the hydraulic system of civil aircraft to give some references for the relevant researchers.
\end{abstract}

\section{Introduction of Hydraulic System of Civil Aircraft}

Civil aircraft hydraulic system is a complex integrated system of redundancy and high power. It has the features of high power, small volume, light weight, quick response and high accuracy. Therefore, in many important areas of metallurgy industry, engineering machinery, aerospace, shipbuilding and other has been widely used. Hydraulic system is always in control and power transmission in all kinds of equipment and systems. With the hydraulic system to develop in the direction of fast, high power, high accuracy, hydraulic systems and equipment are more and more functional and more and more complex. The working strength is more and more heavy correspondingly.

The development of the aircraft hydraulic system mainly towards high voltage, high power and highly integrated development and aircraft actuation system developed with the development of technology, especially modern electrical introduced to make the aircraft control system development is rapid, already by purely mechanical, hydraulic development to electrohydraulic combined with stage and electrical part occupies the proportion continues to increase. In order to ensure the reliability and safety of any aircraft hydraulic system, the hydraulic system is composed of the redundancy and the large power system. Energy system can guarantee to provide the needs of aircraft hydraulic system pressure, flow and the necessary electrical energy, should also guarantee that no matter which energy system problems. The rest of the standby system can guarantee the safety of aircraft landing. The system of users includes the flight control system, landing gear and reverses thrust system, system under the pressure of stablewhich belongs to the part of the function of the aircraft system.

\section{Relevant Parameters of Reliability}

Reliability refers to the ability to complete the required functions when the product is in the specified conditions orsome provisions. Reliability analysis must be based on probability theory, using the necessary parameters of the integration of the probability of occurrence. Different from the general probability, the hydraulic system reliability is a probability function with time 
information, which will produce the corresponding changes according to the time. In order to facilitate the analysis of the reliability of the system, we first need to introduce some relevant parameters:

Failure Rate.Failure rate is the failure probability in the next unit time when the product is normal in the previous t time. It can be formulated as:

$$
\lambda(t)=\frac{\Delta n(t)}{N-n(t)}(1)
$$

The failure rate is generally divided into three parts. The first part along with the time increase will decline; the second part is almost flat; and the third part is a rising curve. The characteristics similar to human death curve. Each of the components may be one of the sections, the general hydraulic reliability calculation using the same part of the calculation.

Reliability.Reliability is also a function of time, which refers to the probability measure of system reliability. It must be completed in a specified time. With the use of time increases, the reliability decreases. The difference between the failure rate and the failure rate is that it refers to the long time failure probability, and the failure probability tends to be zero. The relationship between failure rate and reliability is:

$$
R(t)=e^{-\int_{0}^{1} \lambda(t) d t}(2)
$$

Failure Probability Density.It referred to the unreliable degree of probability density distribution function. Although the parameters will not eventually need to be obtained, the other parameters can deduct. However,the general calculationcannot be found in the documents. In fact, it is consistent with the actual information theWeibull distribution.

\section{Analysis of Reliability Hydraulic System of Civil Aircraft}

Flow Reliability. Aircraft take off when the needs of the traffic is generally relatively large, the need for aircraft landing when the traffic is relatively small, which is required to run the aircraft and the aircraft is related to the actuator. And when the aircraft problems, and will need to meet the large flow conditions. When the EDP cannot be competent for the flow of work, EMP need to meet the additional aircraft traffic function; when the system cannot meet the flow of work, PTU need to open to meet the signal mutual compensation function. Redundant design is the use of multiple sets of systems which have a set of problems through the fault monitoring to be removed or isolated. Redundant design can significantly improve the reliability of the system, so that it can continue to work in the case of failure. The disadvantage is that the cost is high, the control model of the system is complex, and it has a certain limit to the improvement of the system reliability. The introduction of redundant units will inevitably lead to additional costs, in addition to the increase in manufacturing costs, the use and maintenance costs will be increased. The improvement of task reliability is to increase the cost of manufacturing and maintenance costs. It shows a lot of technical development. Many technology produced on the military, and then transferred to civilian use, such as servo valve, a hydraulic booster is the first in the aviation application and then transferred to civilian, believe that redundant design will also be used in many departments. Energy saving design using new components, new technology to achieve energy saving hydraulic system can reduce the system installed power and failure rate. Such as the use of new energy conversion components hydraulic transformer, the hydraulic transformer cannot be lost to the load need to adjust the flow and pressure. The application of hydraulic transformer to the hydraulic system not only brings a 
significant reduction in the power of the system, but also opens up a new way to reduce the energy consumption of the system and simplify the structure of the hydraulic system.

LogicReliability.In order to meet the overall reliability of the aircraft, hydraulic system reliability to meet the flow control, but also need to meet other logical reliability. The hydraulic system is mainly to meet the needs of aircraft control, such as lifting, direction and spoiler after moving. Logic reliability is mainly to meet the state analysis of EDP, which mainly includes pressure, temperature and so on. In order to carry on the analysis of the whole system, we first need to analyze whether the state of the system is normal or not. Under normal condition, the system pressure is needed to satisfy a certain quantity range. We need to meet when the pressure is higher than 24 (X) psi, said the system is working properly; always to less than 1800psi, said the pressure is too low, after the failure of the need to meet higher than 2400psi to say that the system to restore the normal. The rest of the time does not mean that the system error, can meet the correct 2100psi parameters. Work in the seabed, underwater, pollution and other special environment of the hydraulic system to carry out environmental design. In the literature, the platform system which can adjust the ship hull is adapted to the marine environment, and has practical significance to the hydraulic components and systems. The simplified design can improve the basic reliability of the product. Hydraulic system should try to use the pump, valve to reduce the pipeline connection. The modular design can reduce the number of components and their mutual connection.

\section{Prediction of Reliability Hydraulic System of Civil Aircraft}

The development of hydraulic system of civil aircraft in our country is still in the initial stage, and the reliability of the technology needs to be verified, in which the hydraulic system is the key to the success or failure of the whole system. In order to ensure the reliability and stability of the whole system, it is very necessary to predict the reliability of hydraulic system, and it has important practical significance. Reliability prediction is to estimate the reliability of the whole system based on the reliability of the components of the system. This is a from small to big, from local to the whole process, its significance is as a design decision basis, in new product development and design stage, in case of lack of reliability data by fuzzy forecast as a guide, the product reliability prediction, conducive to development work carried out smoothly. In order to obtain high reliability, the hydraulic system must have high reliability and high reliability. Aircraft hydraulic system accuracy requirement is high, but the hydraulic circuit is simple, which electro-hydraulic proportional valve control signals for opening and closing, so as to control the system pressure is the key element of the system, its reliability decide the reliability of the whole system, carries on the forecast analysis can initially predict the system reliability, and through to improve the reliability of the hydraulic components to improve the reliability of the hydraulic system. Due to the hydraulic system itself is fuzzy, so in the research of reliability introduced fuzzy mathematics theory and the hydraulic components of fuzzy and fuzzy reasoning, this is more in line with the working state of the system, reliability prediction of trust value is also greatly improved.

\section{Test of Reliability Hydraulic System of Civil Aircraft}

Aircraft hydraulic development environment to verify the system test platform can be effective of different models fly hydraulic system simulation and verification different pressure levels, different layout types of aircraft hydraulic system principle, characteristics of load flow, pressure pulsation of the pump source, load control, hydraulic control logic and so on many aspects of ground experiments and analysis. Reliability is very important for aircraft hydraulic system, so it is 
widely used in system design, in order to ensure the reliability of aircraft hydraulic system. Due to the reasons for the project schedule, the experimental platform has not been fully completed. The system lacks the key EDP, fuel tank components, resulting in the current stage of the system cannot complete the experimental verification. This chapter will be in front of the analysis in the foundation as far as possible in the existing conditions to carry out various experiments and to validate the system meets the design requirements, the performance parameter test system and verify the design of the hydraulic control logic is valid. Now mainstream models of hydraulic systems are using 3000psi, but high pressure is the future trend of development, so it is necessary to analyze the pressure pulsation characteristics of the pump source under high pressure. The pressure fluctuation peak front system technical agreement does not exceed 4.2MPa, and strive to peak level $\mathrm{E}$ to $1.7 \mathrm{MPa}$. In the experiment, the work pressure of the two levels of $26 \mathrm{MPa}$ and $20 \mathrm{MPa}$ is selected, and the pressure fluctuation of EMP under the same working pressure is studied. The results are shown in Figure 1.

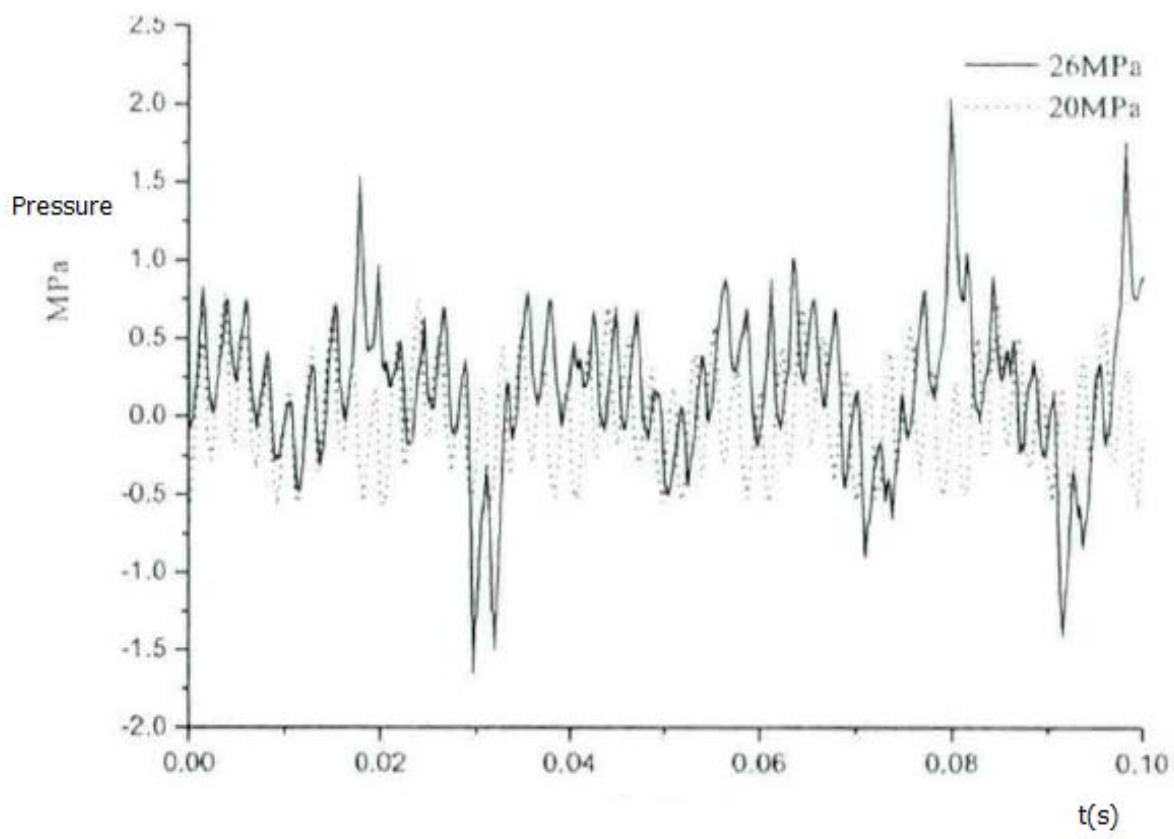

Figure 1.Pulsation comparison under different pressure

When the work pressure amplitude is increased and also in 26mpa to peak value of the pressure pulsation of the system is about 3.5 MPa. When the work pressure is 20MPa peak value of the pressure pulsation is about 1.2 MPa to design requirements. The high pressure of aircraft hydraulic system is a great challenge for hydraulic components and seals. Design at the outlet of the pump source with a length of $1 \mathrm{~m}$ hose, hose test on fruit source pressure fluctuation elimination. In the experiment work pressure 26mpa, at 3000rpm hose before and after the pressure pulsation. The results are shown in Figure 2. 


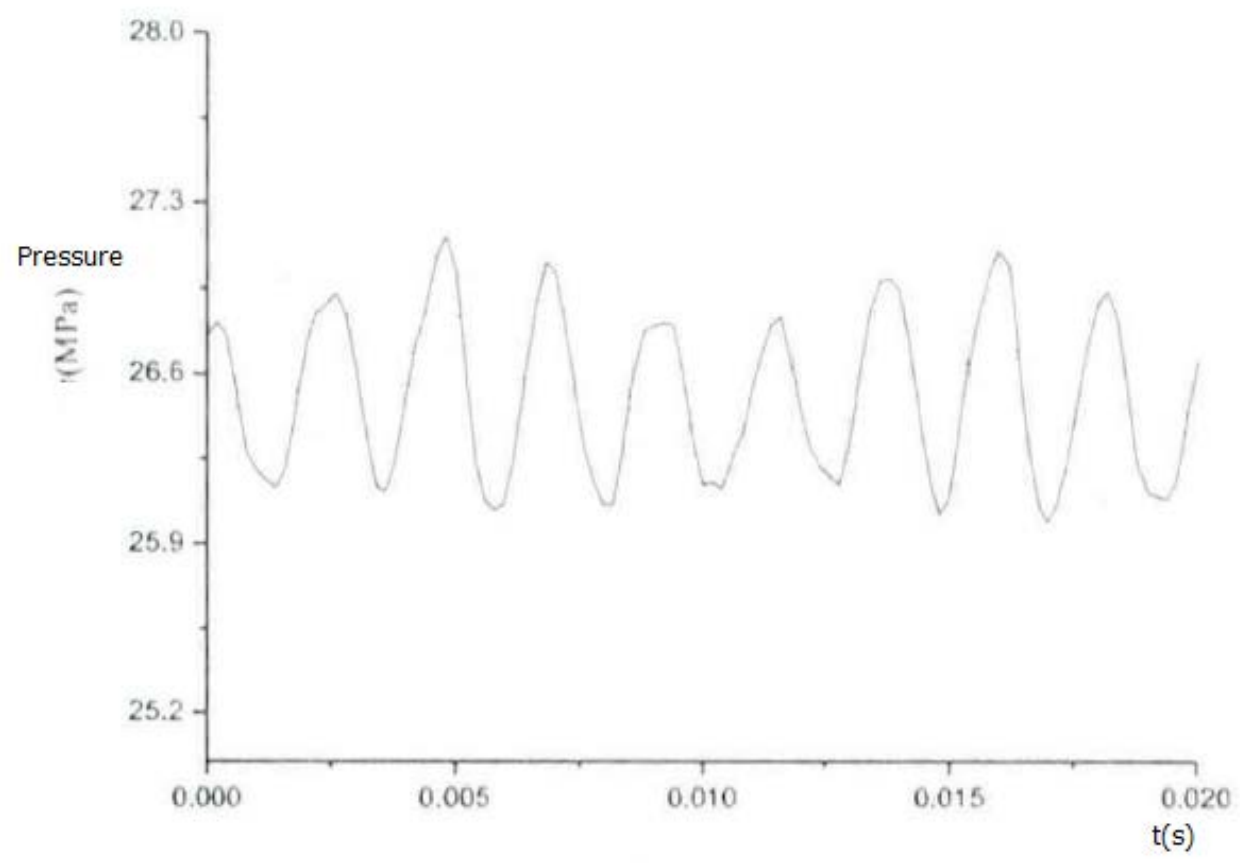

Figure 2.Pulsation comparison between hose or not

We can see from the figure that the hose has a certain degree of attenuation. It is proved that the system source outlet plus the hose to the pressure pulsation has a certain inhibitory effect. Through experiment on pressure fluctuation of the system, we can see the system working pressure increase fruit source pressure fluctuation will increase, which is unfavorable factors for the system and needs to weaken.

\section{References}

[1]Wang Kai, Zhao TianFei, Zhu Ying,Chinese Hydraulics \& Pneumatics, Vol. 39 (2015) No 8,p.20-25

[2]ZhaoJingyi, YaoChengyu, Hydraulics Pneumatics \& Seals, Vol. 26 (2006) No 3,p.50-52

[3] Lu Jianping, Zhou Chunhua, Machine Tool \& Hydraulics, Vol. 30 (2002) No 4,p.143-14

[4] Zhang Qiang, Li Xianghui, Ma Min, Shanxi Metallurgy, Vol. 43(2016) No 2,p.53-56

作者：陈江涛，刘瑞

单位: 中航通飞研究院有限公司

邮寄地址：广东省珠海市金湾区航空产业园中航通飞研究院有限公司 陈江涛（收） 邮编：519040

联系电话： 18926916712 